Voix et Images

\title{
Sortie 21 de Gilles Raymond : entre le Moulin (de la Domtar) et la Maison (ancestrale)
}

\section{Renald Bérubé}

Volume 17, numéro 3 (51), printemps 1992

Paul-Marie Lapointe

URI : https://id.erudit.org/iderudit/200986ar

DOI : https://doi.org/10.7202/200986ar

Aller au sommaire du numéro

Éditeur(s)

Université du Québec à Montréal

ISSN

0318-9201 (imprimé)

1705-933X (numérique)

Découvrir la revue

Citer cet article

Bérubé, R. (1992). Sortie 21 de Gilles Raymond : entre le Moulin (de la Domtar) et la Maison (ancestrale). Voix et Images, 17(3), 536-541.

https://doi.org/10.7202/200986ar d'utilisation que vous pouvez consulter en ligne.

https://apropos.erudit.org/fr/usagers/politique-dutilisation/ 


\title{
Sortie 21 de Gilles Raymond: entre le Moulin (de la Domtar) et la Maison (ancestrale)
}

\section{Renald Bérubé, Université du Québec à Rimouski}

\author{
À Danyelle Morin, \\ du Camp littéraire Félix
}

Chrysanthème se détourne du père, elle fait de nouveau face au paysage. Devant eux, à leurs pieds, la forteresse de la Domtar se dresse au bord du fleuve, là où la rivière Jacques-Cartier vient se fondre dans les eaux immobiles du Saint-Laurent. La cheminée crache son gros nuage gris. Même à cette distance, on peut entendre la rumeur des machines qui grondent à l'abri des murs.

Gilles Raymond, Sortie $21^{1}$

Au bout d'un moment, le petit roi sort à son tour. Deux pas, il hésite: ce qu'il voit lui semble sorti tout droit d'un récit d'épouvante, véritable transcription du registre des histoires extraordinaires; au premier coup d'œil sur le bâtiment, une insupportable tristesse pénètre le King.

Ibid., p. 144

Car le King n'aime pas se raconter et par là retourner sur ses pas.

Ibid., p. 148

De même que, vous le savez depuis un moment déjà, lecture et écriture ont inextricablement partie liée, l'une se mêlant des affaires 
de l'autre et vice versa, de même réalité et fiction entretiennent des liens tout aussi palimpsestueux que ceux décrits par Gérard Genette s'agissant des rapports écriture-lecture. Ainsi on dira, tel fait étant pour le moins étonnant, que la réalité dépasse la fiction, ou que l'on se croirait dans un roman. Symétriquement et inversement, il pourra nous arriver de dire à telle personne qui a tendance à exagérer (à fabuler?), qu'elle ne devrait pas prendre ses rêves pour des réalités, qu'* on n'est pas dans un roman. ni "au cinéma. Ces formules du langage courant, jouant de la comparaison, disent tout à la fois des ressemblances et des différences; elles ont le mérite de souligner en tout cas, nolens volens, que réalité et fiction, si elles sont bien distinctes mais parentes, sont aussi bien parentes mais distinctes. Voilà.

Cela étant, vous n'êtes pas exagérément surpris (ce qui implique que vous l'êtes tout de même un peu) quand, ayant achevé votre deuxième lecture de Sortie 21 de Gilles Raymond au mois d'août 1991 et vous souvenant très nettement de ce passage qui se situe une dizaine de pages avant la fin du roman:

- Si ça peut te donner une idée de ce qui s'en vient... À l'heure actuelle, y'a trente hommes de métier au Moulin [de la Domtar, à Donnacona] et la compagnie envisage que dans un an, un an et demi, y restera même pas assez de travail pour dix d'eux autres! (p. 265)

- quand, donc, lectures du roman et mémoire de celles-ci réagissant à la stimulation, vous lisez dans le quotidien Le Soleil du jeudi 12 septembre 1991, sur cinq colonnes à la (page) B-10, le titre suivant: - Domtar a besoin de 88 millions \$ pour assurer sa relance à Donnacona ", le début de l'article signé Dominic Hardy se lisant ainsi:

DONNACONA - Plus de 500 personnes ont lancé un S.O.S., hier, $\cdot$ à Donnacona, afin d'assurer l'avenir de l'usine de papier journal de Domtar, le plus important employeur privé de la région de Portneuf. Mais il faudra au moins $\mathbf{8 8}$ millions \$ pour assurer sa relance.

Depuis l'automne 1989, Donnacona a perdu environ 250 emplois industriels. Avec la menace qui plane sur l'usine qui emploiera désormais $\mathbf{5 0 0}$ personnes [...] .

Accompagnant l'article, une photo sur laquelle, devant une banderolle affirmant "On veut vivre à Donnacona ", se trouvent, entre autres, le maire de la ville et le président de la CSN.

Vous n'êtes pas exagérément surpris; car Sortie 21 a des intentions réalistes/engagées assez nettes, et la quatrième de couverture du roman vous a rappelé ce que vous saviez déjà : l'auteur est né en 1951 à Donnacona, il habite dorénavant le Bas-Saint-Laurent où il a été très actif tout autant dans la Coalition Urgence rurale qu'au Salon du livre 
de Rimouski. La page * Du même auteur * précédant le roman remet en mémoire, quant à elle, que Gilles Raymond a publié Un moulin, un village, un pays chez VLB en 1981 et Donnacona (récit historique) en 1990. Rien d'exagéré dans la surprise, alors, mais force vous est de reconnaître que s'il arrive à la réalité de dépasser la fiction, il arrive tout aussi bien à la réalité de * copier * la fiction. Mais que veut dire "la réalité copie la fiction " quand la fiction, de toute évidence, s'inspire de la réalité? Peut-être ceci: que l'écriture de fiction est la plus merveilleuse lectrice de toutes les manifestations du réel.

Comment dire, sans qu'il y paraisse trop, qu'il faudrait ici résumer, minimalement, la diégèse de Sortie 21 , alors même que l'idée ne vous sourit guère puisque l'entreprise vous semble impossible? En courtcircuitant ainsi, si on vous le permet, nécessité et impossibilité: Sortie 21 raconte l'histoire de Pierrot Usher, fils de Welly et Jeanne. Welly travaille à la Domtar de Donnacona depuis toujours ou à peu près, dans cette ville à employeur unique ou à peu près, où les fils suivent les traces de leurs pères ou à peu près et deviennent à leur tour employés du Moulin. Mais les temps ont changé, depuis le temps qu'ils s'imitaient: il n'y a plus guère d'emplois nouveaux à la Domtar et Pierrot s'impatiente, le chômage et le BS ne constituant pas un avenir. Pour accélérer les choses, Welly, ancien président de l'Union, accepte de devenir foreman au Moulin, emploi qu'il avait toujours refusé (p. 95); cependant, Pierrot va se donner un autre projet d'existence: rénover la Maison ancestrale des Usher située au bout de la sortie 21 et la vendre ensuite à profit. Mais la Maison où s'est passée sa jeune enfance évoque tous les cauchemars du monde pour Welly, alors que le Moulin 'ne dit plus rien à un Pierrot "écœuré pour de bon: il n'a même plus envie que la Domtar l'appelle» (p. 106). À la fin du roman, le Moulin semble bien n'offrir qu'un avenir de peau de chagrin technologique; la Maison, quant à elle, dévorera, dans un accident de voiture, Pierrot et ses amis qui venaient entreprendre les rénovations. Où donc se situe l'avenir, où donc se situe la sortie 21 , celle qui mène vers le "XXI" siècle" ainsi que l'indique la quatrième de couverture; entre la réfection du passé et les sirènes (du Moulin) de la technologie?

Ce que ce résumé réussit à dire: que Sortie 21 est un roman à visée sociale qui dénonce sinon la ville inhumaine du moins la situation captive dans laquelle se retrouve la population des villes à employeur presque unique. Que la relation Welly-Pierrot n'est pas 
sans évoquer la relation Édouard-Joseph dans Un simple soldat de Marcel Dubé et que le Welly de Sortie 21 n'est pas sans en rappeler un autre, le Willy Loman de la Mort d'un commis voyageur d'Arthur Miller. Ce que ce résumé réussit mal à dire: que le roman de Gilles Raymond présente la "situation captive " de Donnacona dans un contexte très actuel qui soulève d'une part la question du vieillissement de la population, d'autre part celles du chômage, de la drogue et des gangs chez une jeunesse désœuvrée condamnée au BS. Que Sortie 21 est aussi un roman où l'action de type réaliste fourmille, multipliant les épisodes, occupant l'avant-scène et la majeure partie de l'espace du texte. En quelque sorte, Sortie 21 pourrait se lire comme un mixte du Cassé de Jacques Renaud et du Matou d'Yves Beauchemin. Et pourtant.

Et pourtant, le personnage le plus attachant et le plus réussi du roman est peut-être le petit roi, le King, propriétaire de "la Brasserie du King, refuge des égarés. (p. 19). Le King, ému qu'on l'appelle Jacques ainsi qu'il se nomme (p. 183), enfant de l'orphelinat (p. 86, 148) qui rêve d'une belle grande maison ancestrale de style victorien située à Cap-Santé (p. 74). Maison où il se retrouvera à son grand étonnement puisqu'elle est celle de la docteure Johanne Gagnon du CLSC où il va faire soigner Pierrot. Et la docteure Gagnon aimera le King, endossera et lui fera comprendre le sens de l'entreprise qu'il mène auprès des jeunes: «Si tu les aides pas, Jacques... Si tu les aides pas, toi, qui va les aider?» (p. 232). Mais le King, ainsi que le souligne la troisième épigraphe de ce texte, "n'aime pas se raconter et par là retourner sur ses pas *.

Sortie 21 est divisée en trois parties: - La chute de la maison Usher* (p. 7-114, chap. 1-21), *Le secret de la maison Usher * (p. 115223, chap. 22-40), "Pas de sursis pour la maison Usher" (p. 225-274, chap. 41-45). Les première et troisième parties sont précédées d'une épigraphe, celle de la première partie se lisant ainsi:

Il me semblait être sur la limite de la compréhension sans pouvoir comprendre; comme les gens qui sont sur le bord du souvenir, et qui cependant ne parviennent pas à se rappeler.

Extrait provenant des Histoires extraordinaires d'Edgar Allan Poe, du -Double assassinat de la rue Morgue " plus précisément. Or Poe est aussi l'auteur de "La chute de la maison Usher": on aura deviné d'où 
vient le patronyme principal de Sortie 21 et pourquoi Welly craint tant la Maison ancestrale (crainte que le lecteur, par ailleurs, éprouve un peu de mal à partager, même s'il en est souvent question). Qui plus est, l'épigraphe précédant la troisième partie, provenant d'Alexis ou le Traité du vain combat de Marguerite Yourcenar, se lit ainsi:

... on dit que les vieilles maisons contiennent toujours des fantômes; je n'en ai jamais vu, et pourtant j'étais un enfant craintif. Peut-être je comprenais déjà que les fantômes sont invisibles, parce que nous les portons en nous-mêrmes.

Réseau épigraphique auquel il faut encore ajouter ceci: au centre de Sortie 21 ou presque, au chapitre 21, ce roman de Bosco que lit Pierrot sur les recommandations de sa sœur Chrysanthème, roman qui le décide à entreprendre la rénovation de la Maison ancestrale:

Tout est là: l'héritage, ce jeune imbécile qui décide, malgré toutes les faiblesses par lesquelles son caractère semble si proche du sien, de braver les obstacles. L'héritage familial... pourquoi n'y a-t-il pas songé plus tôt! (p. 107).

Tel un hypotexte en abyme au cœur de Sortie 21, le Malicroix d'Henri Bosco, dont les deux premières phrases, le nom Malicroix devenant simplement M..., sont textuellement citées. Et l'on sait que le fantastique ou l'extraordinaire ont aussi la part belle dans certaines œuvres de Bosco - retour à Poe, donc.

Vous vous dites alors ceci, votre deuxième lecture du roman de Gilles Raymond bien achevée: Sortie 21 se déroule pour ainsi dire selon deux intentions, deux points de vue parallèles, deux registres, hésite entre le Moulin et la Maison. Il y a d'une part le roman réaliste et engagé qui constate, qui décrit comme de l'extérieur et avec fébrilité les événements quotidiens, leurs causes et leurs conséquences immédiates; il y a d'autre part cet autre roman, celui que veut nous faire lire le réseau épigraphique ou le Malicroix en abyme, róman plus intériorisé de l'héritage et des fantômes invisibles que nous portons en nous-mêmes. Mais comment dire ces fantômes invisibles et cet héritage si, tel le King selon le narrateur, on "n'aime pas se raconter et par là retourner sur ses pas *? Il ne faut pas s'étonner alors que la lecture inopinée de Malicroix par Pierrot et l'influence décisive que cette lecture exercera sur lui soient racontées en deux (trop?) brèves pages tout au plus.

Sortie 21 a donc surtout privilégié la veine réaliste, le Moulin si l'on peut dire; et vous vous prenez à le regretter un peu. Car il y a ces passages de la veine héritage ou Maison, moments où le récit est plus intériorisé et qui se révèlent magnifiques: le voyage en autobus (le 
conducteur se nomme Malicroix) de la mère Usher (p. 57-62), les brefs souvenirs du père Usher (p. 144-145), les réflexions du King (chap. 34), entre autres. Cela étant, vous relisez cette autre phrase du roman: "Paysages intérieurs, les maisons ancestrales ont quelque chose à dire au King...* (p. 176), phrase qui devrait bien apporter son contrepoids à celle de la page 148 et obliger un jour le King à *se raconter - davantage; et vous relisez aussi la scène terrible/merveilleuse sur laquelle se termine Sortie 21 . Vous vous dites alors que quand on sait créer un personnage comme le King et quand on sait marier, ainsi que dans la scène finale, réalisme et fantômes invisibles, tous les espoirs sont permis. Et vous attendez avec confiance la prochaine * sortie * romanesque de Gilles Raymond.

1. Gilles Raymond, Sortie 21, Montréal, Québec/Amérique, coll. - Littérature d'Amérique , $1990,276 \mathrm{p}$. 